International Journal of Bifurcation and Chaos, Vol. 23, No. 1 (2013) 1350003 (11 pages)

(C) World Scientific Publishing Company

DOI: $10.1142 / \mathrm{S} 021812741350003 \mathrm{X}$

\title{
A TWO-PARAMETER METHOD FOR CHAOS CONTROL AND TARGETING IN ONE-DIMENSIONAL MAPS
}

\author{
DANIEL FRANCO \\ Departamento de Matemática Aplicada, \\ Universidad Nacional de Educación a Distancia, \\ Apartado de Correos 60149, 28080 Madrid, Spain \\ dfranco@ind.uned.es \\ EDUARDO LIZ* \\ Departamento de Matemática Aplicada II, \\ Universidad de Vigo, E. I. Telecomunicación, \\ Campus Marcosende, 36310 Vigo, Spain \\ eliz@dma.uvigo.es
}

Received September 23, 2011; Revised November 24, 2011

\begin{abstract}
We investigate a method of chaos control in which intervention is proportional to the difference between the current state and a fixed value. We prove that this method allows to stabilize the most usual one-dimensional maps used in discrete-time models of population dynamics about a globally stable positive equilibrium. From the point of view of targeting, this technique is very flexible, and we show how to choose the control parameter values to lead the system towards the desired target. Another important feature of this control scheme in the ecological context is that it can be designed to prevent the risk of extinction in models with the so-called Allee effect. We provide a useful geometrical interpretation, and give some examples to illustrate our theoretical results.
\end{abstract}

Keywords: One-dimensional maps; chaos control; global stability; population dynamics; Allee effect.

\section{Introduction}

Control strategies aiming to stabilize chaotic systems about fixed points or periodic orbits should exhibit a number of good features. To list some of them, the method should be easy to implement, the control action should not be too strong, and the controlled system should drive most solutions to the stabilized equilibrium or periodic orbit. Such properties have been discussed for several methods of control; for example, for the proportional feedback method (PF) [Güémez \& Matías, 1993; Liz, 2010a; Braverman \& Liz, 2012; Carmona \& Franco, 2011], and for the prediction-based control (PBC) [de Sousa Vieira \& Lichtenberg, 1996; Ushio \& Yamamoto, 1999; Polyak, 2005; Liz \& Franco, 2010]. Depending on the related problem, some other aspects are of special interest; for example, if the method is applied in the context of population dynamics, one can seek to stabilize the system about a high population level (e.g. in exploited populations), or to a low population level (e.g. in the control of plagues). Another important aspect in the

*Author for correspondence 
framework of population control is trying to avoid the risk of extinction, in particular, preventing the so-called Allee effects, which are defined as a decline in individual fitness at low population size, and can result in critical thresholds below which populations crash to extinction [Courchamp et al., 2008]. For a further discussion about the role of chaos control in ecology, see [Solé et al., 1999].

Let us consider a simple one-dimensional difference equation

$$
x_{n+1}=f\left(x_{n}\right),
$$

where $f: I \rightarrow I$ is a continuous function defined on a real interval $I$. This equation generates a simple discrete dynamical system, whose orbits are the solutions of (1), that is, sequences $\left\{x_{n}\right\}_{n \geq 0}$ constructed by recurrence using (1), starting at some initial condition $x_{0} \in I$. The above mentioned control methods are based on the introduction of an external parameter, which can be controlled to some extent. For example, methods based on proportional feedback consist in adding or removing a percentage $\gamma$ of the state variable, while in $\mathrm{PBC}$ methods, the control is proportional to the difference between the current state $x_{n}$ and a prediction of a future state $f^{k}\left(x_{n}\right)$, where $f^{k}$ denotes, as usual, the $k$ th iteration of $f$.

If we want to improve the features of control techniques, a good approach is considering methods depending on more than one parameter, in such a way that a kind of optimal control (depending on the desired goal) can be determined by a clever combination of those parameters. A recent attempt in this direction is the target-oriented control (TOC) introduced in [Dattani et al., 2011] in the context of population dynamics governed by (1). The control method writes

$$
\begin{aligned}
x_{n+1} & =f\left(x_{n}+c\left(T-x_{n}\right)\right) \\
& =f\left(c T+(1-c) x_{n}\right) .
\end{aligned}
$$

Notice that two new parameters were added to the equation; in [Dattani et al., 2011], $c$ is called the control and $T$ is referred to as the target. As noticed in [Dattani et al., 2011], if we fix $T=0$, then the control scheme (2) becomes the usual PF control method. For $T \neq 0$, it can be seen as a combination of the PF method and the modified constant feedback method (MCF) introduced in [Wieland, 2002]. An important observation is that, when $T$ is chosen as an equilibrium of (1), then $T$ is an equilibrium of (2) for every value of $c$; thus, an equilibrium of the uncontrolled equation can be stabilized using (2). This is a common property with the PBC method and the delayed feedback control (DFC) introduced by Pyragas [1992]. A comparison between TOC and PBC methods can be found in [Dattani et al., 2011].

We show that when $T$ is different from the equilibrium, the TOC method becomes a powerful tool from the point of view of targeting since we are able to explain the response of the system to control, in such a way that appropriate parameter values can be chosen depending on the pursued goal. In particular, we provide rigorous proofs of some numerical observations given in [Dattani et al., 2011] in this direction.

Another important remark is that, if the range of values of $c$ is restricted to the interval $[0,1]$, and $T \in I$, where $I$ is the domain of definition of $f$, it is ensured that, for each $x_{0} \in I$, there is a unique solution $\left\{x_{n}\right\}$ of (2) defined for all $n \geq 0$. The reason is that $c T+(1-c) x_{n}$ is a convex combination of $x_{n}$ and $T$. In the context of population dynamics, this is a very important issue because it ensures that a permanent system remains permanent after control. Values of $c$ greater than 1 can induce catastrophe bifurcations, driving the population to a sudden extinction in many cases; see Fig. 1 in [Dattani et al., 2011]. Thus, we will restrict our study to $c \in[0,1]$ and $T \in I$.

The paper is organized as follows: in Sec. 2, we introduce a modified target-oriented control method (MTOC), which has interest in itself and, besides, it will help to investigate the TOC method. In Sec. 3, we provide a geometric interpretation of the equilibria of both TOC and MTOC methods, and prove a result of stabilization. Section 4 is focused on unimodal maps with a unique positive equilibrium; we prove a result of global stabilization, and discuss how the size of the stabilized equilibria changes when control is applied, depending on the two parameter values. Section 5 is devoted to unimodal maps modeling populations that undergo the so-called Allee effect; we show that the control methods discussed here allow us to both stabilize the system and prevent extinction. Finally, we summarize our conclusions and suggest some directions for future research in Sec. 6.

\section{A Modified TOC Method}

Let us notice that scheme (2) assumes that the control acts before the dynamical system; in the 
context of population dynamics, this means that control intervention is applied before reproduction [Dattani et al., 2011]. If we assume, on the contrary, that intervention occurs after reproduction, a modified target-oriented control method (MTOC) can be considered in the form

$$
\begin{aligned}
y_{n+1} & =f\left(y_{n}\right)+c\left(T-f\left(y_{n}\right)\right) \\
& =c T+(1-c) f\left(y_{n}\right) .
\end{aligned}
$$

Let us fix a value of $T \in I$. Denoting $\phi_{c}(x)=$ $c T+(1-c) x$, it is clear that the change of variables $y_{n}=\phi_{c}\left(x_{n}\right)$ transforms (2) into (3). This means that both difference equations are topologically conjugate. Indeed, if we denote by $g_{c}(x):=$ $f\left(\phi_{c}(x)\right)$ and $G_{c}(x)=\phi_{c}(f(x))$ the maps defining the right-hand side of (2) and (3), respectively, then the relationship $\phi_{c} \circ g_{c}=G_{c} \circ \phi_{c}$ holds. This fact implies that Eqs. (2) and (3) share the same dynamics from a topological point of view (see, e.g. [Alligood et al., 1996, Section 3.3]). For example, $\phi_{c}$ maps orbits of (2) to orbits of (3), and $O=\left\{x_{1}, \ldots, x_{p}\right\}$ is a $p$-periodic orbit of $(2)$ if and only if $\phi_{c}(O)=\left\{\phi_{c}\left(x_{1}\right), \ldots, \phi_{c}\left(x_{p}\right)\right\}$ is a $p$-periodic orbit of (3); moreover, if $f$ is differentiable then the multipliers coincide and therefore the stability properties of $O$ and $\phi_{c}(O)$ are the same. In particular, $K_{c}$ is an equilibrium of (2) if and only if $P_{c}=\phi_{c}\left(K_{c}\right)$ is an equilibrium of (3), and they have the same stability properties. The following relationship between $K_{c}$ and $P_{c}$ will be useful:

$$
K_{c}=g\left(K_{c}\right)=f\left(\phi_{c}\left(K_{c}\right)\right)=f\left(P_{c}\right) .
$$

Finally, we notice that $P_{c}$ is globally asymptotically stable for (3) if and only if $K_{c}=f\left(P_{c}\right)$ is globally asymptotically stable for (2).

\section{Equilibria}

It is easy to provide a geometric interpretation of the equilibria of (2) and (3), and how they change as $c$ ranges from 0 to 1 , for a fixed value of $T$. Since in this paper we will have in mind population models, we will assume the following condition holds:

(A1) $f:[0, b] \rightarrow[0, b](b=\infty$ is allowed) is continuously differentiable, $f(0)=0$, and $f(x)>0$ for all $x \in(0, b)$.

In most usual models from population dynamics, (A1) holds for maps defined on $[0, \infty)$ such as the Ricker map $f(x)=x e^{r(1-x)}, r>0$, and the generalized Beverton-Holt map $f(x)=r x /\left(1+x^{\gamma}\right)$, $r>0, \gamma>0$. In other cases, such as the quadratic function $f(x)=r x(1-x), r \in(0,4]$, the map is defined on a compact interval $[0,1]$.

A positive real number $P_{c}$ is an equilibrium of (3) if it satisfies

$$
P_{c}=c T+(1-c) f\left(P_{c}\right) \Leftrightarrow f\left(P_{c}\right)=\frac{P_{c}-c T}{1-c} .
$$

Thus, if $c \in[0,1)$, the equilibria of (3) are given by the intersections between the graph of $f$ and the line

$$
y=\frac{x-c T}{1-c} .
$$

In the limit case $c=1$, the line is defined by $x=T$. Notice the line $(6)$ has slope $1 /(1-c)$, and passes through the point $(T, T)$. Thus, the family of lines whose intersection with the graph of $f$ give the positive equilibria of (3) are obtained by rotating continuously a line passing through the point $(T, T)$ from the line $y=x$ to the vertical line $x=T$. For each $c \in(0,1)$, the positive equilibria $P_{c}$ of (3) are given by the projection over the horizontal axis of the intersection points of $f$ with the line defined by (6).

Now it follows from (4) that if $P_{c}$ is a positive equilibrium of (3), then $K_{c}=f\left(P_{c}\right)$ is a corresponding equilibrium point of (2). This means that the positive equilibria of (2) are the projection over the vertical axis of the intersection points of $f$ with the line defined by (6). In Figs. 1 and 2, we show how to visualize the equilibria of (2) and (3) for a unimodal map with a unique positive equilibrium $K$ in cases $T>K$ and $T<K$, respectively.

An interesting remark is that, under some mild assumptions, both (2) and (3) have at least a positive equilibrium, and it becomes asymptotically stable if a sufficiently strong control is implemented. In view of the previous discussion, it is enough to consider the MTOC method.

Lemma 1. Assume that (A1) holds and there exists a positive constant $M$ such that $f(x) \leq x$ for all $x \geq M$. Let $T \in(0, b]$ be arbitrarily fixed, and define

$$
B=\max \{M, T\}, \quad C=\max _{x \in[0, B]}\left\{\left|f^{\prime}(x)\right|\right\} .
$$

Then there exists at least an equilibrium $P_{c}$ of (3) in $(0, B]$ for every $c \in(0,1)$. Moreover, $P_{c}$ is asymptotically stable for all $c \in(1-1 / C, 1)$. 


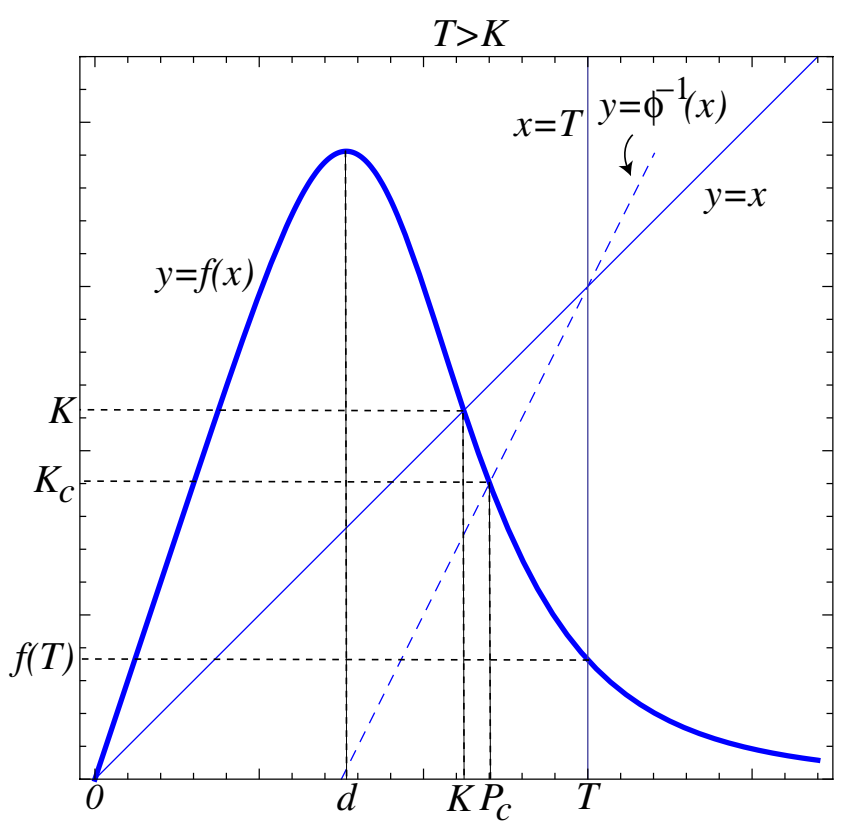

Fig. 1. Representation of a unimodal map $f$ with a positive fixed point $K$. The fixed points $K_{c}$ of the TOC method (2) with $T>K$ are a decreasing function of $c$, and take values between $K_{0}=K$ and $K_{1}=f(T)$. For the MTOC method (3), the fixed points are an increasing function of $c$, ranging between $P_{0}=K$ and $P_{1}=T$.

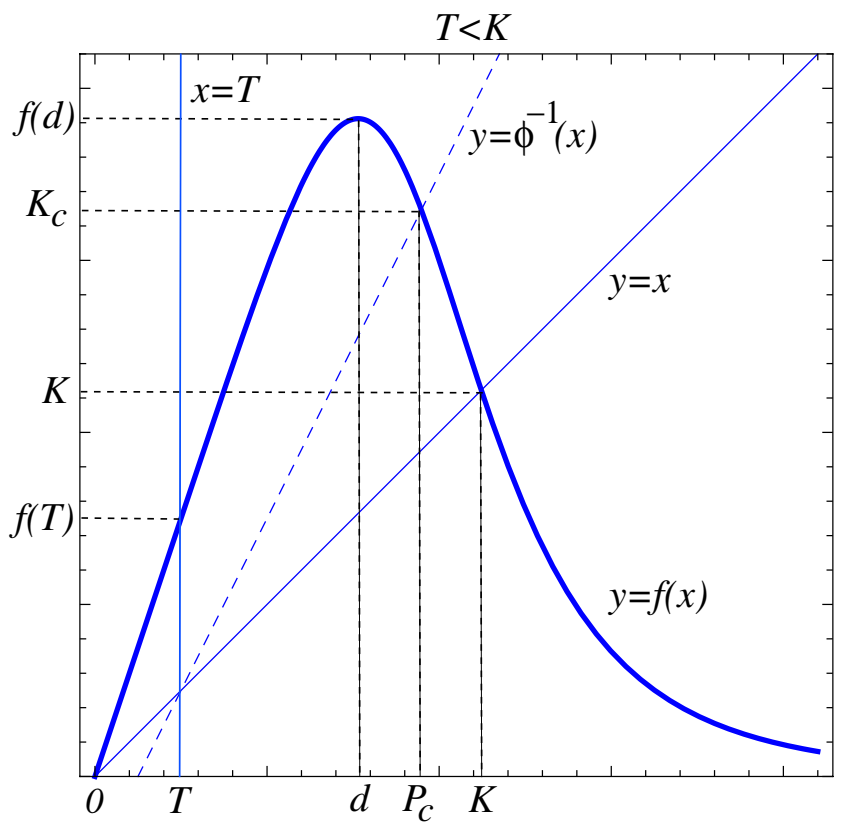

Fig. 2. Representation of a unimodal map $f$ with a positive fixed point $K$. The fixed points $K_{c}$ of the TOC method (2) with $T<d<K$ are a unimodal function of $c$, first increasing between $K_{0}=K$ and $f(d)$, and then decreasing from $f(d)$ to $K_{1}=f(T)$. For the MTOC method (3), the fixed points are a decreasing function of $c$, ranging between $P_{0}=K$ and $P_{1}=T$.
Proof. Recall that the equilibria of (3) are the fixed points of $G_{c}(x)=\phi_{c}(f(x))=c T+(1-c) f(x)$. Assume that $c \in(0,1)$. Notice that $G_{c}(0)=c T>0$ and

$G_{c}(B)=c T+(1-c) f(B) \leq c B+(1-c) B=B$.

Thus, either $G_{c}(B)=B$ or there is a fixed point of $G_{c}$ in $(0, B)$.

Finally, from the definition of $C$, it is clear that if $P_{c} \in[0, B]$ is an equilibrium of (3) then, for all $c \in(1-1 / C, 1)$,

$$
\left|G_{c}^{\prime}\left(P_{c}\right)\right|=(1-c)\left|f^{\prime}\left(P_{c}\right)\right| \leq(1-c) C<1,
$$

implying that $P_{c}$ is asymptotically stable.

In the following sections, we provide much sharper stability results for some classes of maps usually employed in population dynamics.

\section{Overcompensatory Models}

In this section, we consider maps $f$ satisfying the following assumptions, besides (A1):

(A2) $f$ has only two non-negative fixed points $x=0$ and $x=K>0, f(x)>x$ for $0<x<K$, and $f(x)<x$ for $x>K$.

(A3) $f$ has a unique critical point $d<K$ in such a way that $f^{\prime}(x)>0$ for all $x \in(0, d), f^{\prime}(x)<0$ for all $x>d$, and $f^{\prime \prime}(x)<0$ on $(0, d)$.

The graph of a map satisfying (A1)-(A3) is a so-called overcompensatory curve [Clark, 1990, Chapter 7]. This class of functions is often employed in discrete-time population models, and it includes the Ricker map, the Hassel map, and the generalized Beverton-Holt map considered in [Dattani et al., 2011], among others. For more discussions about these assumptions, see [Liz, 2010a; Liz \& Franco, 2010].

For the PF method (that is, TOC with $T=0$ ), a result of global stabilization for maps satisfying (A1)-(A3) was proved in [Liz, 2010a]. We next demonstrate how such result can be extended for the general TOC and MTOC methods, with an arbitrary $T \in(0, b)$.

Theorem 1. Assume that (A1)-(A3) hold, and let us fix $T \in(0, b)$. Then both (2) and (3) have a unique positive equilibrium for each $c \in[0,1)$. Moreover, there exists $c_{1} \in[0,1)$ such that the positive equilibria of (2) and (3) are asymptotically 
stable for all $c \in\left(c_{1}, 1\right)$. If the following additional assumption is required:

(A4)

$$
\begin{aligned}
& (S f)(x)<0 \text { for all } x \neq d, \text { where } \\
& \qquad(S f)(x)=\frac{f^{\prime \prime \prime}(x)}{f^{\prime}(x)}-\frac{3}{2}\left(\frac{f^{\prime \prime}(x)}{f^{\prime}(x)}\right)^{2}
\end{aligned}
$$

is the Schwarzian derivative of $f$,

then the positive equilibria of (2) and (3) are globally asymptotically stable for all values of $c$ for which they are locally asymptotically stable.

Proof. Based on the conjugacy relationship discussed in Sec. 2, it is enough to prove the result for MTOC. We recall that (3) can be rewritten as

$$
y_{n+1}=G_{c}\left(y_{n}\right),
$$

where $G_{c}(x)=\phi_{c}(f(x))=c T+(1-c) f(x)$.

It follows from Lemma 1 that there is at least a positive equilibrium of $(3)$ for every $c \in(0,1)$.

It is clear that $G_{c}$ satisfies (A3) for all $c \in[0,1)$ because $G_{c}^{\prime}(x)=(1-c) f^{\prime}(x)$ and $G_{c}^{\prime \prime}(x)=(1-$ c) $f^{\prime \prime}(x)$ for all $x \in[0, b]$. Thus $G_{c}$ is concave and increasing on $(0, d)$, and decreasing on $(d, b)$. These properties imply that $G_{c}$ cannot have more than one positive fixed point (otherwise, by the Mean Value Theorem, there should be a point $x_{*}$ such that $G_{c}^{\prime}\left(x_{*}\right)=1$ and $\left.G_{c}^{\prime \prime}\left(x_{*}\right) \geq 0\right)$.

Applying Lemma 1 again, we know that there is a value $c_{1} \in(0,1)$ such that $\left|G_{c}^{\prime}\left(P_{c}\right)\right|<1$ for all $c \in\left(c_{1}, 1\right)$, and hence $P_{c}$ is locally asymptotically stable for $c>c_{1}$.

Next, since $G_{c}=\phi_{c} \circ f$, and $\left(S \phi_{c}\right)(x)=0$ for all $x \in \mathbb{R}$, Theorem 2.1 in [Singer, 1978] ensures that $\left(S G_{c}\right)(x)=(S f)(x)<0$ for all $x \neq d$. Since $G_{c}$ is unimodal, it follows that $P_{c}$ is globally asymptotically stable for all values of $c$ for which it is locally asymptotically stable (see, e.g. [Liz et al., 2003, Proposition 3.3]).

Some remarks are in order.

\section{Remark 4.1}

- We recall that many usual maps employed in discrete-time models of population dynamics satisfy the technical assumption (A4); see, e.g. [Singer, 1978; Schreiber, 2001; Thunberg, 2001].

- If $T>0$ then the positive equilibrium $K_{c}$ of (2) does exist for all values of $c \in(0,1)$. This is a difference with the limit case $T=0(\mathrm{PF}$ method), where the equilibrium only exists for $c \in\left(c_{1}, 1-1 / f^{\prime}(0)\right)$. This means that if $T>0$ and conditions (A1)-(A4) hold, then the TOC method is not only stabilizing, but also, extinction is not possible if $c \in(0,1)$.

- Assuming that $f^{\prime}(K)<-1$ [otherwise, $K$ is asymptotically stable for the uncontrolled Eq. (1)], the positive equilibrium $P_{c}$ of (3) becomes asymptotically stable after a periodhalving bifurcation when

$$
(1-c) f^{\prime}\left(P_{c}\right)=-1 .
$$

Thus, in the statement of Theorem 1 we can choose $c_{1}$ as the supremum of $c \in(0,1)$ for which (7) holds.

Although the dynamical properties of (2) and (3) are equivalent, there is an important difference between them regarding the response to control in the size of the stabilized equilibrium. This fact has important implications when choosing one of the two control methods either in the management of exploited populations or in control of plagues. From the geometric interpretation of the equilibria of (3), it is clear that if (A2) and (A3) hold then the equilibrium $P_{c}$ increases with $c$ if $T>K$, and decreases if $0 \leq T<K$. Of course, if $T=K$, the equilibrium remains constant for any value of $c \in[0,1]$. See Figs. 1 and 2 .

For the TOC method (2), the equilibrium $K_{c}$ decreases with $c$ if $T>K$, increases with $c$ if $d<$ $T<K$, and it is first increasing and then decreasing if $0 \leq T<d$. Notice that $P_{c}$ ranges monotonically from $P_{0}=K$ to $P_{1}=T$, while $K_{c}$ ranges from $K_{0}=K$ to $K_{1}=f(T)$, but it does it in a monotone way only if $T>d$.

Based on the results of Theorem 1 and the previous discussion, we may generalize another feature of the PF method stated in [Liz, 2010a] to the TOC scheme. Indeed, if $0 \leq T<d$ then Eq. (1) can be globally stabilized with $(2)$ at about any value between $f(T)$ and $f(d)=\max \{f(x): x \in(0, d)\}$. See Fig. 2.

Next we illustrate our results with a case of study.

Example 4.1. Consider the generalized BevertonHolt map

$$
f(x)=\frac{3 x}{1+x^{6}}
$$

which is chaotic and satisfies (A1)-(A4). The global maximum of $f$ is attained at $d \approx 0.76472$, with a 


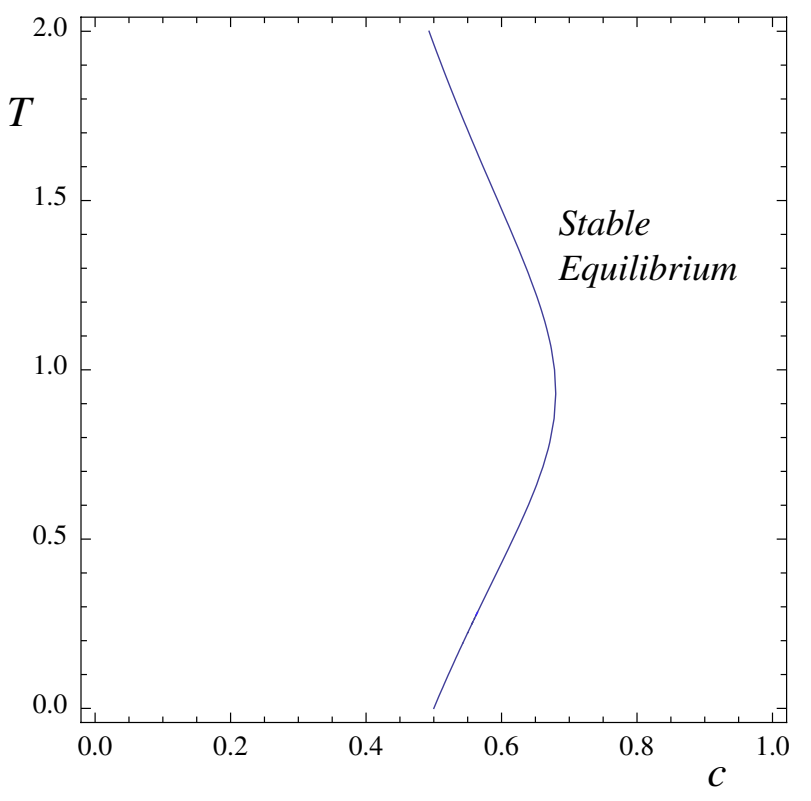

Fig. 3. Border of asymptotic stability for (2) and (3) in the plane $(c, T)$ for the generalized Beverton-Holt map $f(x)=3 x /\left(1+x^{6}\right)$.

value of $f(d) \approx 1.91181$. The unique fixed point of $f$ is $K=2^{1 / 6} \approx 1.12246$.

From Theorem 1, we can ensure that, for each $T>0$ and $c \in(0,1)$, the control schemes (2) and (3) have a unique positive equilibrium, and that it is globally stabilized for a sufficiently large value of the control parameter $c$. In Fig. 3, we plot the border of the asymptotic stability region for both methods in the plane of parameters $(c, T)$, with $c \in(0,1)$ and $T \in(0,2)$. It reveals that the strength of the control parameter $c$ necessary to stabilize the system should be greater for intermediate values of $T$.

A very important issue that one should take into account before applying these control methods is the size of the equilibrium about which the system is stabilized. As shown in Fig. 4, the response to control is very different for both methods and for the different choices of the target parameter $T$. As discussed in this section, when MTOC is applied, the size of the positive equilibrium is a monotone function of $c$, decreasing if $T<K$, and increasing if $T>K$. If $T=K$ then the equilibrium remains constant for any value of $c$. The response is completely different if the intervention takes place before reproduction. In this case, some counterintuitive effects are observed. For example, if $x_{0} \leq f(d)$, then a value of $T$ greater than $f(d)$ means that population is supplied at each intervention, but this results in a decrease of the population size; this phenomenon can be interpreted as a form of the paradox of enrichment [Rosenzweig, 1971]. Conversely, low values of $T$ mean that part of the population is usually removed when control is applied, but the response

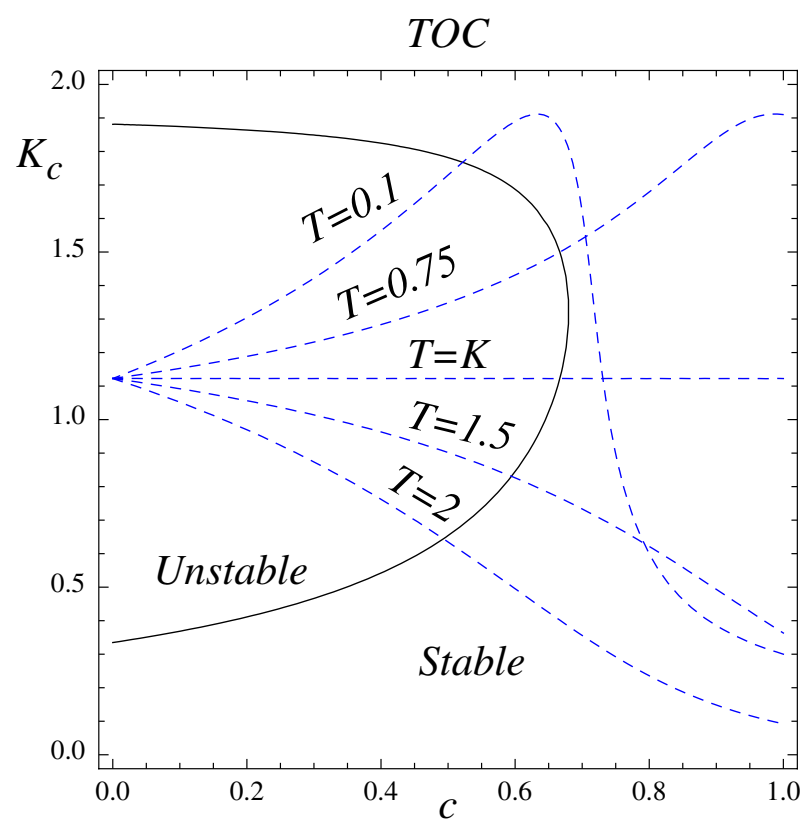

(a)

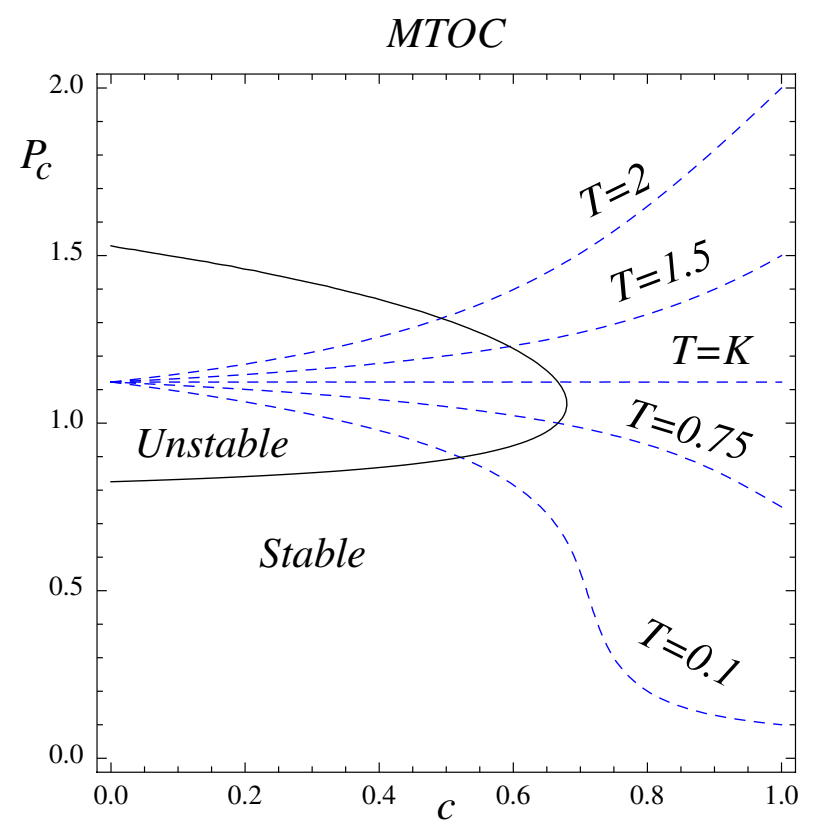

(b)

Fig. 4. (a) Representation of the positive equilibria of (2) for different values of $T$ in the plane $\left(c, K_{c}\right)$. (b) Representation of the positive equilibria of (3) for different values of $T$ in the plane $\left(c, P_{c}\right)$. In both cases, the solid line is defined by Eq. (7), and represents the border of asymptotic stability. We use the generalized Beverton-Holt map $f(x)=3 x /\left(1+x^{6}\right)$. 
of the system is an increase in its size; this phenomenon has been termed the hydra effect [Abrams, 2009; Liz \& Ruiz-Herrera, 2012]. It is important to notice that the geometric interpretation given in the previous section allows us to know the effect of the control intervention depending on the relative position of the target parameter $T$ with respect to the natural equilibrium of the system and the critical point at which the reproduction rate is maximum. For example, if we use the TOC method, values of $T$ smaller than the critical value $d$ allow to reach the maximum production rate $f(d)$; if we choose $T$ close to zero, then this value is attained with a smaller control strength, but then the equilibrium drops suddenly to very low values; however, a value of $T$ close to $d$ requires a stronger control intervention to stabilize the system about $f(d)$, but this strategy reduces the risk of leading the system to undesired low values; compare the cases $T=0.1$ and $T=0.75$ in Fig. 4(a).

\section{Models with Allee Effect}

In the previous sections, we have shown that both TOC and MTOC methods are a good tool to stabilize a positive equilibrium in usual unimodal maps with a unique positive equilibrium. Not only is global asymptotic stability achieved, but also the method is very flexible from the point of view of targeting. However, Theorem 1 does not apply to models with Allee effect. This effect is crucial in conservation and management, and it is attracting much interest in recent years [Courchamp et al., 2008; Eskola \& Parvinen, 2010]. Most usual control methods fail to prevent Allee effects; for example, the CF control method induces an Allee effect, rather than preventing it [Parthasarathy \& Sinha, 1995; Schreiber, 2001; Liz, 2010b]. On the other hand, both PBC and PF methods are not suitable for this type of models (see [Liz, 2010a; Liz \& Franco, 2010]). Although Theorem 3 in [Carmona \& Franco, 2011] goes in this direction, its applicability is limited.

In this section, we demonstrate that TOC offers good chances to tackle the problem of control in models with Allee effect, both avoiding this undesirable effect, and stabilizing the system about a positive equilibrium. Although we will restrict our discussion to the TOC method, everything can be easily applied to the MTOC method too.

Throughout this section, we will assume that $f$ satisfies (A1). Instead of (A2) and (A3), we will require the following condition:

(A5) $f$ has several non-negative fixed points $0=$ $\kappa_{0}<\kappa_{1}<\cdots<\kappa_{m}$, and there is a unique critical point $d<\kappa_{m}$ in such a way that $f^{\prime}(x)>0$ for all $x \in(0, d)$, and $f^{\prime}(x)<0$ for all $x>d$.

Our first result concerns the case $T \geq d$.

Lemma 2. Assume that (A1) and (A5) hold. If $T \geq d$ then there exists $c_{2} \in(0,1)$ such that (2) has a unique positive equilibrium for all $c \in\left(c_{2}, 1\right)$. Moreover, we can take

$$
c_{2}=\max _{0 \leq x \leq \kappa_{m-1}}\left\{\frac{f(x)-x}{f(x)-T}\right\} .
$$

Proof. First, let us notice that, since $\kappa_{m-1}<d<$ $\kappa_{m}$, condition $T \geq d$ implies that $f(x)<T$ for all $x \in\left[0, \kappa_{m-1}\right]$.

Having in mind the geometric interpretation of the equilibria of (2), to ensure that there is only a positive equilibrium, it is enough to prove that

$$
f(x)>\frac{x-c T}{1-c}, \quad \forall x \in\left[0, \kappa_{m-1}\right] .
$$

Since $f(x)-T<0$, the previous inequality is equivalent to

$$
c>\frac{f(x)-x}{f(x)-T}, \quad \forall x \in\left[0, \kappa_{m-1}\right],
$$

that is to say $c>c_{2}$.

Now we can state a theorem for models with Allee effect.

Theorem 2. Assume that (A1) and (A5) hold, and let us fix $T \in(d, b)$. Then there exists $c_{3} \in(0,1)$ such that (2) has a unique positive equilibrium, which is asymptotically stable for all $c \in\left(c_{3}, 1\right)$. Moreover, if (A4) holds, then the positive equilibrium is globally asymptotically stable for all values of $c$ for which it is locally asymptotically stable.

Proof. It is enough to choose $c_{3}=\max \left\{c_{1}, c_{2}\right\}$, where $c_{1}$ is the supremum of $c \in(0,1)$ for which $(7)$ holds, and $c_{2}$ was defined in (8).

When $T \in\left[\kappa_{m-1}, d\right)$, it is still possible to prevent the Allee effect and stabilize the system about a globally stable positive equilibrium. In particular, if we choose $T=\kappa_{m-1}$ and (A1), (A5) hold, 
then $\kappa_{m-1}$ is an equilibrium of (2), and it is globally asymptotically stable for all $c \in\left(c_{4}, 1\right)$, where $c_{4}=\max \left\{M_{1}, M_{2}\right\}$, and

$$
\begin{aligned}
& M_{1}=\sup \left\{\frac{f(x)-x}{f(x)-\kappa_{m-1}}: x \in\left[0, \kappa_{m}\right] \backslash\left\{\kappa_{m-1}\right\}\right\} ; \\
& M_{2}=1-\frac{1}{f^{\prime}\left(\kappa_{m-1}\right)} .
\end{aligned}
$$

Indeed, it is easy to check that $\kappa_{m-1}$ is the only equilibrium of (2) if $c>M_{1}$, and $0<f^{\prime}\left(\kappa_{m-1}\right)<1$ for all $c>M_{2}$. From these facts, and having in mind that $g_{c}(x)=f(c T+(1-c) x)$ is either increasing or unimodal if $T<d$, the global stability of $\kappa_{m-1}$ for $c>c_{4}$ follows.

Remark 5.1. The previous result makes a difference with PBC method. It is easy to check that it is impossible to stabilize a fixed point $K$ of (1) using the PBC scheme

$$
x_{n+1}=c x_{n}+(1-c) f\left(x_{n}\right)
$$

if $c \in(0,1)$ and $f^{\prime}(K)>1$.

Example 5.1. We illustrate the results in this section using a generalization of the quadratic map (see, e.g. [Clark, 1990; Boukal \& Berec, 2002]). Consider the map $f:[0,1] \rightarrow[0,1]$ defined by

$$
f(x)=\frac{27}{4} x^{2}(1-x) .
$$

The map $f$ has two positive fixed points $0<\kappa_{1}<$ $\kappa_{2}$ given by

$$
\begin{aligned}
& \kappa_{1}=\frac{9-\sqrt{33}}{18} \approx 0.180858 ; \\
& \kappa_{2}=\frac{9+\sqrt{33}}{18} \approx 0.819142 .
\end{aligned}
$$

See Fig. 5 for a graphic representation of $f$. The fixed points are the intersections between the graph of $f$ and the line $x=y$.

It is easy to check that (A1) and (A5) hold. The only critical point of $f$ is $d=2 / 3$, and $f(2 / 3)=1$. Hypothesis (A4) also holds, since

$$
(S f)(x)=-\frac{6\left(1-4 x+6 x^{2}\right)}{(2-3 x)^{2} x^{2}}
$$

which is negative for all $x \in(0,1], x \neq d$.

Equation (1) with this function exhibits a strong Allee effect, since $f^{\prime \prime}(0)>0$ [Eskola \& Parvinen, 2010]; moreover, its dynamics is essential

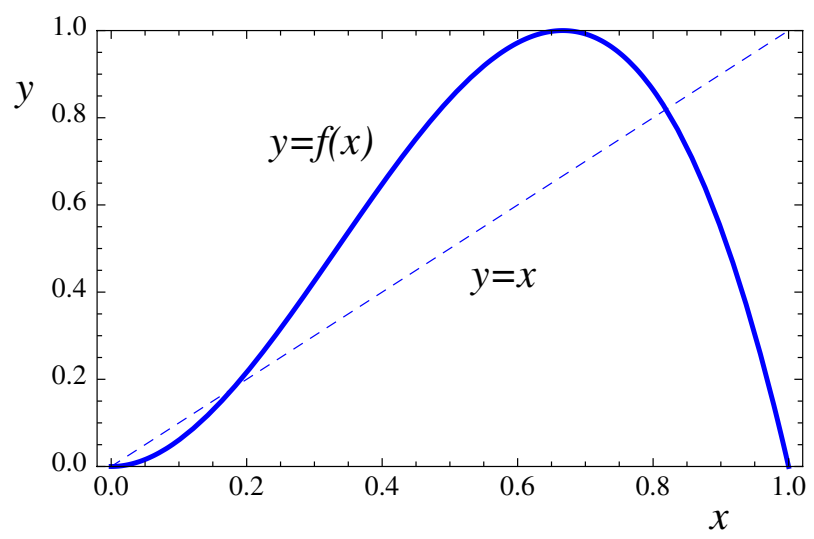

Fig. 5. Representation of the map $f$ defined in (9), which exhibits a strong Allee effect. There are two positive fixed points $\kappa_{1}=(9-\sqrt{33}) / 18, \kappa_{2}=(9+\sqrt{33}) / 18$, which are the intersections between the graph of $f$ and the line $y=x$.

extinction, i.e. for a randomly chosen initial condition, extinction occurs with probability one [Schreiber, 2003].

We apply the control scheme (2) with two different choices of $T: T=d$ and $T=\kappa_{1}$.

In the first case, Theorem 2 can be applied to prove that the controlled Eq. (2) has a globally stable positive equilibrium for all $c>c_{3}$, where $c_{3}=\max \left\{c_{1}, c_{2}\right\}$. In this case,

$$
c_{2}=\max _{0 \leq x \leq \kappa_{1}}\left\{\frac{f(x)-x}{f(x)-d}\right\} \approx 0.0652648
$$

and $c_{1} \approx 0.472728$ is the value at which $f^{\prime}\left(K_{c_{1}}\right)=$ -1 , leading to a period-halving bifurcation. Thus, $c_{3}=c_{1}$. A bifurcation diagram is shown in Fig. 6 .

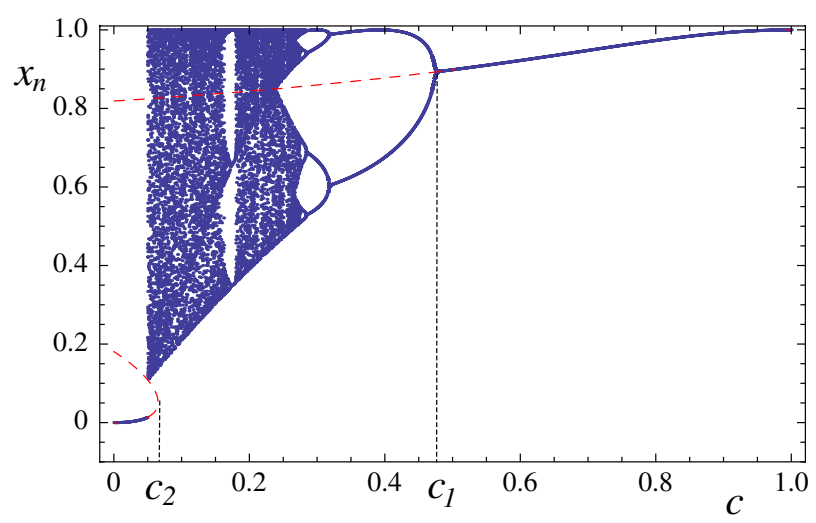

Fig. 6. Bifurcation diagram for the controlled equation $x_{n+1}=f\left(c T+(1-c) x_{n}\right)$, where $f$ is defined in $(9)$, and $T=2 / 3$. A random initial condition was chosen for each value of $c \in(0,1)$. The dashed lines represent unstable equilibria. Notice that there is a unique positive equilibrium for $c>c_{2}$, and it is globally asymptotically stable for $c>c_{1}$. 


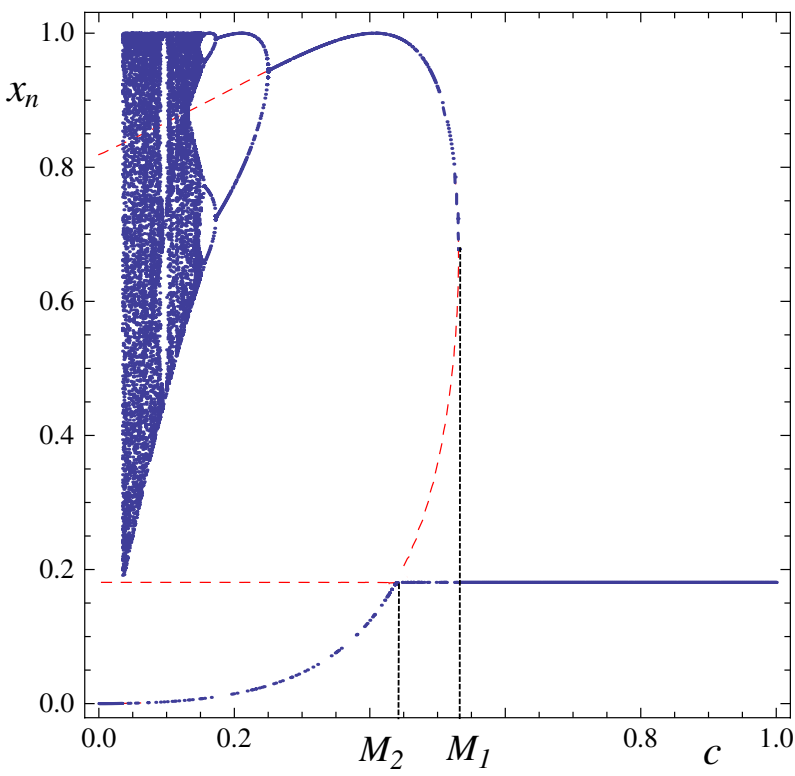

Fig. 7. Bifurcation diagram for the controlled equation $x_{n+1}=f\left(c T+(1-c) x_{n}\right)$, where $f$ is defined in $(9)$, and $T=\kappa_{1} \approx 0.180858$. A random initial condition was chosen for each value of $c \in(0,1)$. The dashed lines represent unstable equilibria. As $c$ is increased, the system ranges from essential extinction to bistability and then to global stability of $\kappa_{1}$.

Notice that the system is stabilized about an equilibrium greater than $\kappa_{2}$, reaching the maximum value $f(d)=1$ at $c=1$.

Next we consider the case $T=\kappa_{1}$. According to our discussion below Theorem $2, \kappa_{1}$ is an equilibrium of (2) for all $c \in(0,1)$, and it is globally asymptotically stable for all $c \in\left(c_{4}, 1\right)$, where $c_{4}=\max \left\{M_{1}, M_{2}\right\}$, and

$$
\begin{aligned}
M_{1} & =\sup \left\{\frac{f(x)-x}{f(x)-\kappa_{1}}: x \in\left[0, \kappa_{2}\right] \backslash\left\{\kappa_{1}\right\}\right\} \\
& \approx 0.531023 ; \\
M_{2} & =1-\frac{1}{f^{\prime}\left(\kappa_{1}\right)} \approx 0.437953 .
\end{aligned}
$$

Thus, $c_{4}=M_{1}$. A bifurcation diagram is shown in Fig. 7. Notice that the system is stabilized about $\kappa_{1}$, for all $c>M_{1}$, after a tangent bifurcation.

\section{Discussion}

In a recent paper, Dattani et al. suggested a method for controlling chaos called target-oriented control (TOC). This method is based on the introduction of two external parameters $c, T$, in such a way that control interventions are proportional to the difference between $T$ and the current state of the system, with a control strength given by $c$. Dattani et al. [2011] gave some numerical results using one-dimensional maps from models of population dynamics, and discussed how the method could be implemented, and analyzed the relationship of TOC with other control schemes.

Our aim in this paper was to provide some rigorous results on the ability of the method to convert chaotic behavior into periodic orbits for a family of one-dimensional maps usually employed in population dynamics. For unimodal maps with negative Schwarzian derivative, like the quadratic and the Ricker maps, we proved that for each choice of the parameter $T$ the controlled equation has a globally attracting positive equilibrium for $c$ large enough (Theorem 1). The method proves to be not only efficient to drive chaotic maps towards a stable equilibrium, but it is also flexible for targeting; that is, a suitable choice of $T$ allows us to carry the system into a desired objective. Moreover, a geometric interpretation of the control given in Sec. 2 is very helpful to choose the appropriate value of $T$. In the context of population dynamics, the TOC method involves interventions at each reproduction period, prior to breeding; we introduced a modified TOC method (MTOC) which acts after reproduction. Although both methods are topologically conjugated (and so they share the same stability properties), the size of the stable equilibrium attained after control is different. We have explained how the equilibrium varies depending on the method and the parameter $T$, increasing in this way the flexibility of control to reach the desired size of the population (a low size in control of plagues or a high size if our aim is to increase production in an exploited population). An interesting remark is that some counterintuitive effects appear if control acts before reproduction; these paradoxical phenomena include the so-called hydra effect (an increase in population size in response to an increasing mortality [Abrams, 2009; Liz \& Ruiz-Herrera, 2012]), and a form of the paradox of enrichment [Rosenzweig, 1971].

It is worth emphasizing that both TOC and MTOC methods can be considered as a generalization of the proportional feedback (PF) control (obtained for $T=0$ ). Since the PF method is still used as a model in ecology (see, e.g. [Yakubu et al., 2011] and its references), the methods investigated in the present paper may provide a new framework 
for control programs in which population can be either reduced or increased through external interventions. In this regard, we showed in Sec. 5 that TOC and MTOC methods work well in population models exhibiting the Allee effect, both avoiding this effect and stabilizing the system about a positive equilibrium. We recall that Allee effects can lead to threshold population densities, below which the population growth is negative, making extinction possible; the most usual cause of Allee effects is the difficulty of finding mates at low population sizes. There has been an increasing interest in the study of these effects in recent years (see [Eskola \& Parvinen, 2010] and references therein).

We point out that prevention of extinction is one of the main motivations for chaos control in ecology [Hilker \& Westerhoff, 2007]. Sometimes, disappearances might occur after transient chaos [Schreiber, 2003]; control of transient chaos in ecology is therefore an important issue that has been addressed in a variety of works, e.g. [Dhamala \& Lai, 1999; Shulenburger et al., 1999]. For new interesting control strategies in this direction, see [Sabuco et al., 2010].

Finally, we feel that this paper is only a first step in making a rigorous study of the properties of TOC and MTOC methods in chaos control problems. An interesting direction for further research is the application of these control techniques to discrete maps of dimension higher than one, such as the Hénon map [Hénon, 1976]. From the point of view of applications in population control, we find it is relevant to study how these methods work when a pulse strategy of interventions is used (generalizing in this way recent work for the PF control [Braverman \& Liz, 2012]), and how robust are the methods in the presence of environmental noise; for some comments in this direction, see the supplementary material to [Dattani et al., 2011].

\section{Acknowledgments}

This research was supported by the Spanish Ministry of Science and Innovation and FEDER, grant MTM2010-14837. The authors thank anonymous referees for their useful comments.

\section{References}

Abrams, P. A. [2009] "When does greater mortality increase population size? The long story and diverse mechanisms underlying the hydra effect," Ecol. Lett. $\mathbf{1 2}, 462-474$.
Alligood, K. T., Sauer, T. \& Yorke, J. A. [1996] Chaos: An Introduction to Dynamical Systems (SpringerVerlag, USA).

Boukal, D. S. \& Berec, L. [2002] "Single-species models of the Allee effect: Extinction boundaries, sex ratios and mate encounters," J. Theoret. Biol. 218, 375-394.

Braverman, E. \& Liz, E. [2012] "Global stabilization of periodic orbits using a proportional feedback control with pulses," Nonlin. Dyn. 67, 2467-2475.

Carmona, P. \& Franco, D. [2011] "Control of chaotic behaviour and prevention of extinction using constant proportional feedback," Nonlin. Anal. Real World Appl. 12, 3719-3726.

Clark, C. W. [1990] Mathematical Bioeconomics: The Optimal Management of Renewable Resources, 2nd edition (John Wiley \& Sons, USA).

Courchamp, F., Berec, L. \& Gascoigne, J. [2008] Allee Effects in Ecology and Conservation (Oxford University Press, USA).

Dattani, J., Blake, J. C. H. \& Hilker, F. M. [2011] "Target-oriented chaos control," Phys. Lett. A 375, 3986-3992.

de Sousa Vieira, M. \& Lichtenberg, A. J. [1996] "Controlling chaos using nonlinear feedback with delay," Phys. Rev. E 54, 1200-1207.

Dhamala, M. \& Lai, Y.-C. [1999] "Controlling transient chaos in deterministic flows with applications to electrical power systems and ecology," Phys. Rev. E 59, 1646-1655.

Eskola, H. T. M. \& Parvinen, K. [2010] "The Allee effect in mechanistic models based on inter-individual interaction processes," Bull. Math. Biol. 72, 184-207.

Güémez, J. \& Matías, M. A. [1993] "Control of chaos in unidimensional maps," Phys. Lett. A 181, 29-32.

Hénon, M. [1976] "A two-dimensional mapping with a strange attractor," Commun. Math. Phys. 50, 69-77.

Hilker, F. M. \& Westerhoff, F. H. [2007] "Preventing extinction and outbreaks in chaotic populations," $\mathrm{Am}$. Nat. 170, 232-241.

Liz, E., Tkachenko, V. \& Trofimchuk, S. [2003] "A global stability criterion for scalar functional differential equations," SIAM J. Math. Anal. 35, 596-622.

Liz, E. [2010a] "How to control chaotic behaviour and population size with proportional feedback," Phys. Lett. A 374, 725-728.

Liz, E. [2010b] "Complex dynamics of survival and extinction in simple population models with harvesting," Theor. Ecol. 3, 209-221.

Liz, E. \& Franco, D. [2010] "Global stabilization of fixed points using predictive control," Chaos 20, 023124.

Liz, E. \& Ruiz-Herrera, A. [2012] "The hydra effect, bubbles, and chaos in a simple discrete population model with constant effort harvesting," J. Math. Biol. 65, 997-1016. 
Parthasarathy, S. \& Sinha, S. [1995] "Controlling chaos in unidimensional maps using constant feedback," Phys. Rev. E 51, 6239-6242.

Polyak, B. T. [2005] "Chaos stabilization by predictive control," Autom. Rem. Contr. 66, 1791-1804.

Pyragas, K. [1992] "Continuous control of chaos by self-controlling feedback," Phys. Lett. A 170, 421428.

Rosenzweig, M. L. [1971] "Paradox of enrichment: Destabilization of exploitation ecosystems in ecological time," Science 171, 385-387.

Sabuco, J., Zambrano, S. \& Sanjuán, M. A. F. [2010] "Partial control of chaotic transients using escape times," New J. Phys. 12, 113038.

Schreiber, S. J. [2001] "Chaos and population disappearances in simple ecological models," J. Math. Biol. 42, 239-260.

Schreiber, S. J. [2003] "Allee effect, extinctions, and chaotic transients in simple population models," Theor. Popul. Biol. 64, 201-209.
Shulenburger, L., Lai, Y.-C., Yalçinkaya, T. \& Holt, R. D. [1999] "Controlling transient chaos to prevent species extinction," Phys. Lett. A 260, 156-161.

Singer, D. [1978] "Stable orbits and bifurcation of maps of the interval," SIAM J. Appl. Math. 35, 260-267.

Solé, R. V., Gamarra, J. G. P., Ginovart, M. \& López, D. [1999] "Controlling chaos in ecology: From discrete maps to individual-based models," Bull. Math. Biol. 61, 1187-1207.

Thunberg, H. [2001] "Periodicity versus chaos in onedimensional dynamics," SIAM Rev. 43, 3-30.

Ushio, T. \& Yamamoto, S. [1999] "Prediction-based control of chaos," Phys. Lett. A 264, 30-35.

Wieland, C. [2002] "Controlling chaos in higher dimensional maps with constant feedback: An analytic approach," Phys. Rev. E 66, 016205.

Yakubu, A.-A., Li, N., Conrad, J. M. \& Zeeman, M.-L. [2011] "Constant proportion harvest policies: Dynamic implications in the Pacific halibut and Atlantic cod fisheries," Math. Biosci. 232, 66-77. 